POS PROCEEDINGS

\title{
Results of radiative and annihilation penguin decays at Belle
}

\section{Dutta (On Behalf of Belle Collaboration)*}

Tata Institute of Fundamental Research, India

E-mail: deepanwita.duttadtifr.res.in

In this paper we report the results of radiative and annihilation penguin B decays at Belle. Penguin processes serve as a sensitive probe to search for physics beyond the Standard Model.

XXIV International Workshop on Deep-Inelastic Scattering and Related Subjects

11-15 April, 2016

DESY Hamburg, Germany

\footnotetext{
* Speaker.
} 


\section{Introduction}

The $b \rightarrow s, d \gamma$ transitions (Figure $\mathbb{W}$ ) are Flavor Changing Neutral Current (FCNC) processes which are forbidden at tree level in the Standard Model (SM) and can only proceed via penguin loops. Penguin processes are sensitive to physics beyond the SM as observables such as branching fraction (BF), CP asymmetry, forward-backward asymmetry, Isospin asymmetry and polarization asymmetry could be modified by the presence of new physics particles in the loops. The decay rates can be expressed by an effective hamiltonian having $\mathscr{Q}_{7}$ term. Thus new physics effect will modify the corresponding Wilson coefficient $\mathscr{C}_{7}$. Thus, precise CP asymmetry and BF measurements of these decays will help to predict or rule out new physics.

In this report, I discuss the recent results of inclusive $\bar{B} \rightarrow X_{s+d} \gamma$, semi-inclusive $\bar{B} \rightarrow X_{s} \gamma$ and exclusive $B^{0} \rightarrow \phi \gamma, B_{s}^{0} \rightarrow \phi \gamma$ and $B_{s}^{0} \rightarrow \gamma \gamma$ modes at Belle. The results reported here are based on $711 \mathrm{fb}^{-1}$ of $\Upsilon(4 \mathrm{~S})$ data and $121.4 \mathrm{fb}^{-1}$ of $\Upsilon(5 \mathrm{~S})$ data collected with the Belle detector [U] at the KEKB []] asymmetric energy B-factory at KEK in Japan.

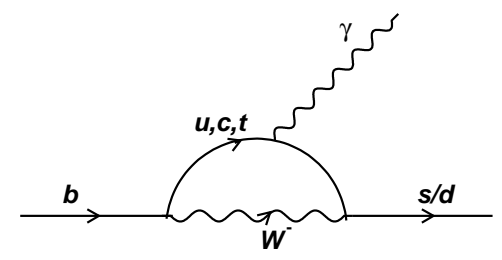

Figure 1: Feynman diagram for $b \rightarrow s, d \gamma$ transitions.

\section{CP asymmetry of $\bar{B} \rightarrow X_{s+d} \gamma$}

The $\mathrm{CP}$ asymmetry $\left(\mathscr{A}_{C P}\right)$ in $\bar{B} \rightarrow X_{s+d} \gamma$ decay is defined as :

$$
A_{C P}\left(\bar{B} \rightarrow X_{s+d} \gamma\right)=\frac{\Gamma\left(\bar{B} \rightarrow X_{s+d} \gamma\right)-\Gamma\left(B \rightarrow X_{\bar{s}+\bar{d}}\right)}{\Gamma\left(\bar{B} \rightarrow X_{s+d} \gamma\right)+\Gamma\left(B \rightarrow X_{\bar{s}+\bar{d}}\right)}
$$

where, $\Gamma\left(\bar{B} \rightarrow X_{s+d} \gamma\right)$ represents the decay rate of the $\bar{B}^{0}$ or $B^{-}$meson into the radiative final state. Charge-conjugate states are implied in this report. The $X_{s+d}$ states includes all possible hadronic final states having a strange or a down quark. The SM predicts $\mathscr{A}_{C P}\left(\bar{B} \rightarrow X_{s+d} \gamma\right)$ to be zero with negligible theoretical uncertainty [B]]. Thus, it serves as a good probe to test for new CP-violating phases. New physics models like SUSY with minimal flavor violation predict $\mathscr{A}_{C P}\left(\bar{B} \rightarrow X_{s+d} \gamma\right)$ upto a level of $+2 \%$ while some other models predict its value to be as high as $10 \%$ [䧃]. Previous $\mathscr{A}_{C P}$ measurements (statistically limited) were done by the CLEO [5] and BaBar [G] collaborations.

In this analysis done in Belle, we have used fully inclusive approach with leptonic tag [ [ $]$ ]. In this approach, only the photon is reconstructed, but the hadronic system $\left(X_{s+d}\right)$ recoiling against the emitted photon is not reconstructed. $X_{s+d}$ includes all accessible final states having a strange or a down quark. This reconstruction method involves huge background but provides a high efficiency. 
To improve the background suppression leptonic tagging is used, i.e., by high energy leptons from the other $B\left(B_{t a g}\right)$ in the event is reconstructed. This method results in reduced signal statistics. Though it is ideal to measure the $\mathrm{BF}$ and $\mathrm{CP}$ asymmetries over the full photon energy $\left(E_{\gamma}\right)$ range, but usually a cut is applied on the $E_{\gamma}$ spectrum to exclude the low energy region as this region is populated with large backgrounds. The photon candidate is required to have an energy $1.7 \mathrm{GeV}<$ $E_{\gamma}<2.8 \mathrm{GeV}$. We require the lepton momentum to be $1.10 \mathrm{GeV}<p_{\ell}^{*}<2.25 \mathrm{GeV}$ in the center of mass $(\mathrm{CM})$ frame. Various loose selection criteria based on track multiplicity, impact parameters, photon energies, numbers of clusters and average cluster energy, polar angles, etc are applied to filter out the beam background events and reduce other backgrounds. Photons from $\pi^{0}$ and $\eta$ are vetoed using some requirements based on photon energy, polar angle and diphoton mass. After the preselection, the dominant background comes from $q \bar{q}$ (continuum) events. These backgrounds are suppressed using event shape variables in Boosted Decision trees. The signal is extracted by subtracting the continuum and the $B \bar{B}$ backgrounds. The continuum background is subtracted using the off-resonance data. $B \bar{B}$ backgrounds are estimated using simulated events. The resulting background subtracted photon energy spectrum is shown in Figure $\square$. $\mathscr{A}_{C P}$ is measured to be $(2.2 \pm 3.9$ (stat. $) \pm 0.9($ syst. $)) \%$ for $E_{\gamma}>2.1 \mathrm{GeV}$.

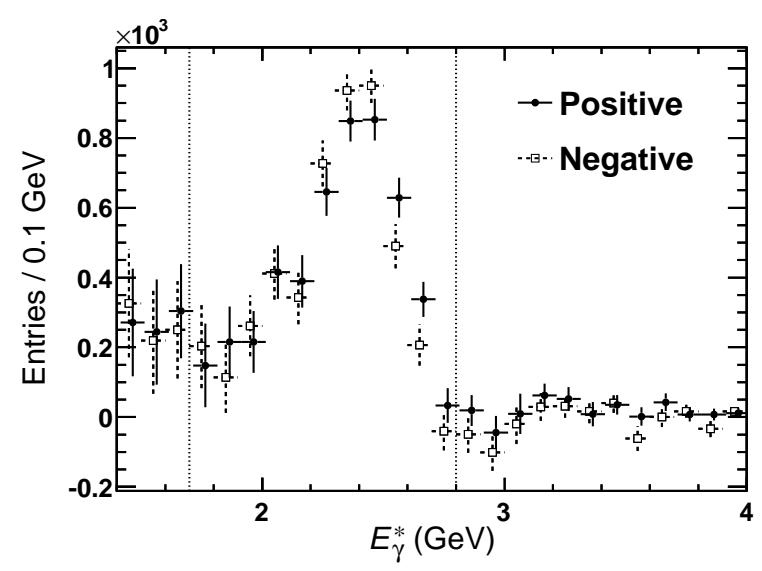

Figure 2: Background subtracted $E_{\gamma}$ spectrum in the CM frame.

\section{Branching Fraction measurement of $\bar{B} \rightarrow X_{s} \gamma$ using the sum of exclusive decays}

The SM prediction of $\bar{B} \rightarrow X_{s} \gamma$ BF at next-to-next leading order is $(3.15 \pm 0.23) \times 10^{-4}$ for $E_{\gamma}>1.6 \mathrm{GeV}$ in the B meson rest frame [ [8]. The world average of its BF is $(3.43 \pm 0.20) \times 10^{-4}[$ [Q] . At Belle, the $\bar{B} \rightarrow X_{s} \gamma$ BF is calculated using semi-inclusive (or sum of exclusive) approach. In this approach, the photon is selected and many exclusive final states (of $X_{S}$ ) are also reconstructed. Events are required to satisfy the selection criteria of one of the exclusive modes. We then sum over the reconstructed exclusive modes. Due to tighter selection criteria there are less backgrounds as compared to fully inclusive case. In semi-inclusive analysis, the recoil hadronic mass spectrum can be measured (instead of the photon spectrum), which can then be converted to an equivalent photon energy spectrum using:

$$
E_{\gamma}^{B}=\frac{M_{B}^{2}-M_{H}^{2}}{2 M_{B}}
$$


This allows for a better measurement of the spectrum shape as the hadronic mass $\left(M_{H}\right)$ resolution can be an order of magnitude better than the photon energy spectrum. At the same time the hadronization error is also suppressed by the reconstruction of as many $B \rightarrow X_{s} \gamma$ states as possible.

In this analysis [ए]] $38 X_{s}$ states were reconstructed. Photon candidates having CM energy $1.8 \mathrm{GeV}<E_{\gamma}^{*}<3.4 \mathrm{GeV}$ are selected. The dominant continuum backgrounds are suppressed using event shape variables in a Neural Network. To veto $\pi^{0}$ coming from $B \rightarrow D(*) \gamma$ decay, D candidates of the major decay modes are reconstructed with combinations of particles used in the $X_{S}$ reconstruction. Events having reconstructed D mass close to the nominal D mass are vetoed. The signal yields are extracted by a maximum likelihood fit to the beam constrained mass $\left(M_{b c}\right)$. To reduce the systematic uncertainty due to $X_{s}$ mass modeling, we divide the data into $19 M_{X_{s}}$ bins in the region $0.6 \mathrm{GeV} / \mathrm{c}^{2}<M_{X_{s}}<2.8 \mathrm{GeV} / \mathrm{c}^{2}$. Figure [ B shows the partial BF as a function of $M_{X_{s}}$. Total BF in $M_{X_{s}}<2.8 \mathrm{GeV} / \mathrm{c}^{2}$ is obtained from the sum of $19 M_{X_{s}}$ bins to be $B\left(\bar{B} \rightarrow X_{s} \gamma\right)=(3.51 \pm 0.17$ (stat. $) \pm 0.33($ syst. $\left.)\right) \times 10^{-4}$. To compare with the theoretical prediction, the experimental result is extrapolated down to $1.6 \mathrm{GeV}$, which introduces model-dependence. The extrapolated BF is $B\left(B \rightarrow X_{s} \gamma\right)=(3.74 \pm 0.18 \pm 0.35) \times 10^{-4}$ which is consistent with the SM prediction within $1.3 \sigma$ and the most precise value till date.

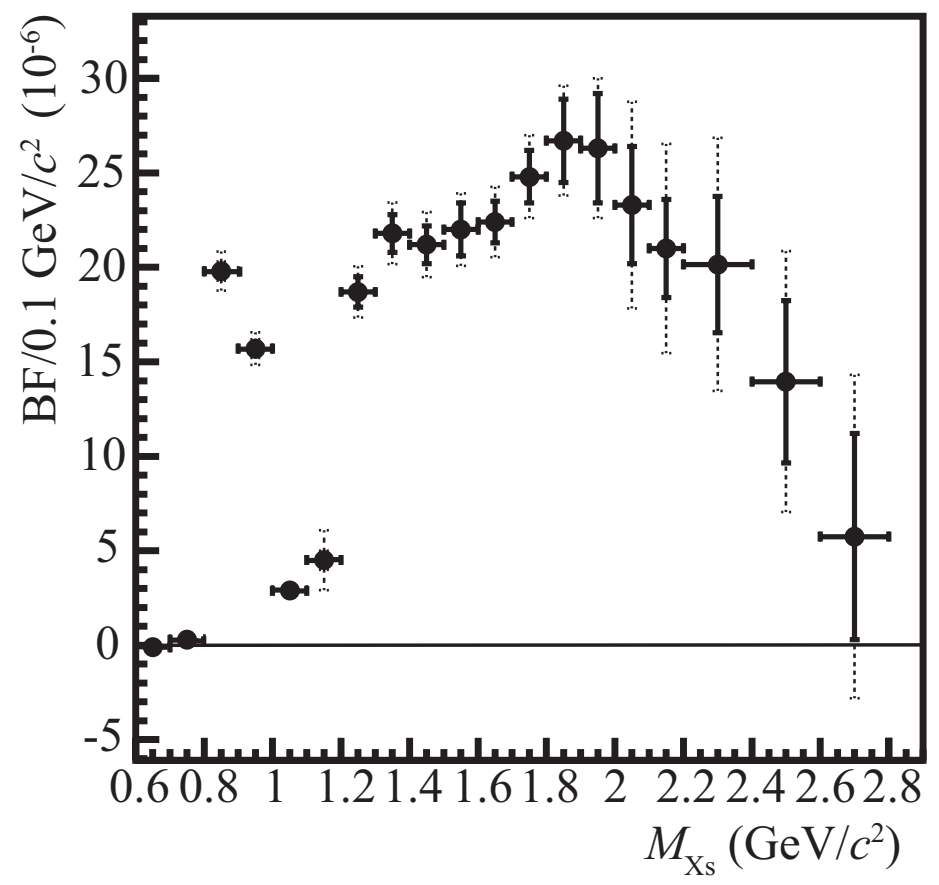

Figure 3: Partial branching fraction as a function of $M_{X_{S}}$.

\section{Search for exclusive modes $B^{0} \rightarrow \phi \gamma, B_{s}^{0} \rightarrow \phi \gamma$ and $B_{s}^{0} \rightarrow \gamma \gamma$}

A $B$-meson decaying into an exclusive final state is reconstructed by measuring the energy and momentum of all long lived decay products $\left(\pi^{ \pm}, K^{ \pm}, e^{ \pm}, \mu^{ \pm}\right.$and $\left.\gamma\right)$ and selecting the intermediate states with certain invariant mass. The exclusive reconstruction method has an advantage of having 
strong kinematic discrimination against the background.

$B^{0} \rightarrow \phi \gamma$ is extremely suppressed in the SM. According to the the SM, its BF lies in the range $\left(10^{-12}-10^{-11}\right)[\mathbb{W}$. Experimentally the $90 \%$ confidence level (CL) upper limit (UL) on its BF is estimated to be $8.5 \times 10^{-7}$ by the Babar experiment [ए2]. $B^{0} \rightarrow \phi \gamma$ mode is reconstructed and various kinematic selection criteria are applied to reduce the backgrounds. Topological variables are fed to the neural network for continuum suppression. A modified neural network output $\left(\mathscr{C}_{N B^{\prime}}\right)$ is calculated and a 4 dimensional maximum likelihood fit involving $M_{\mathrm{bc}}, \Delta E, \cos \left(\theta_{\text {helicity }}\right)$ and $\mathscr{C}_{N B^{\prime}}$ is performed to extract the signal yield. No statistically significant signal $\left(3.4_{-3.8}^{+4.6}\right)$ is observed for the decay $B^{0} \rightarrow \phi \gamma$ i.e., no evidence of $B \rightarrow \phi \gamma$ signal is observed. The 90\% CL UL is estimated to be $1.0 \times 10^{-7}$ [[13]]. Figure $⿴$ t shows the fit projections of $B^{0} \rightarrow \phi \gamma$ analysis.
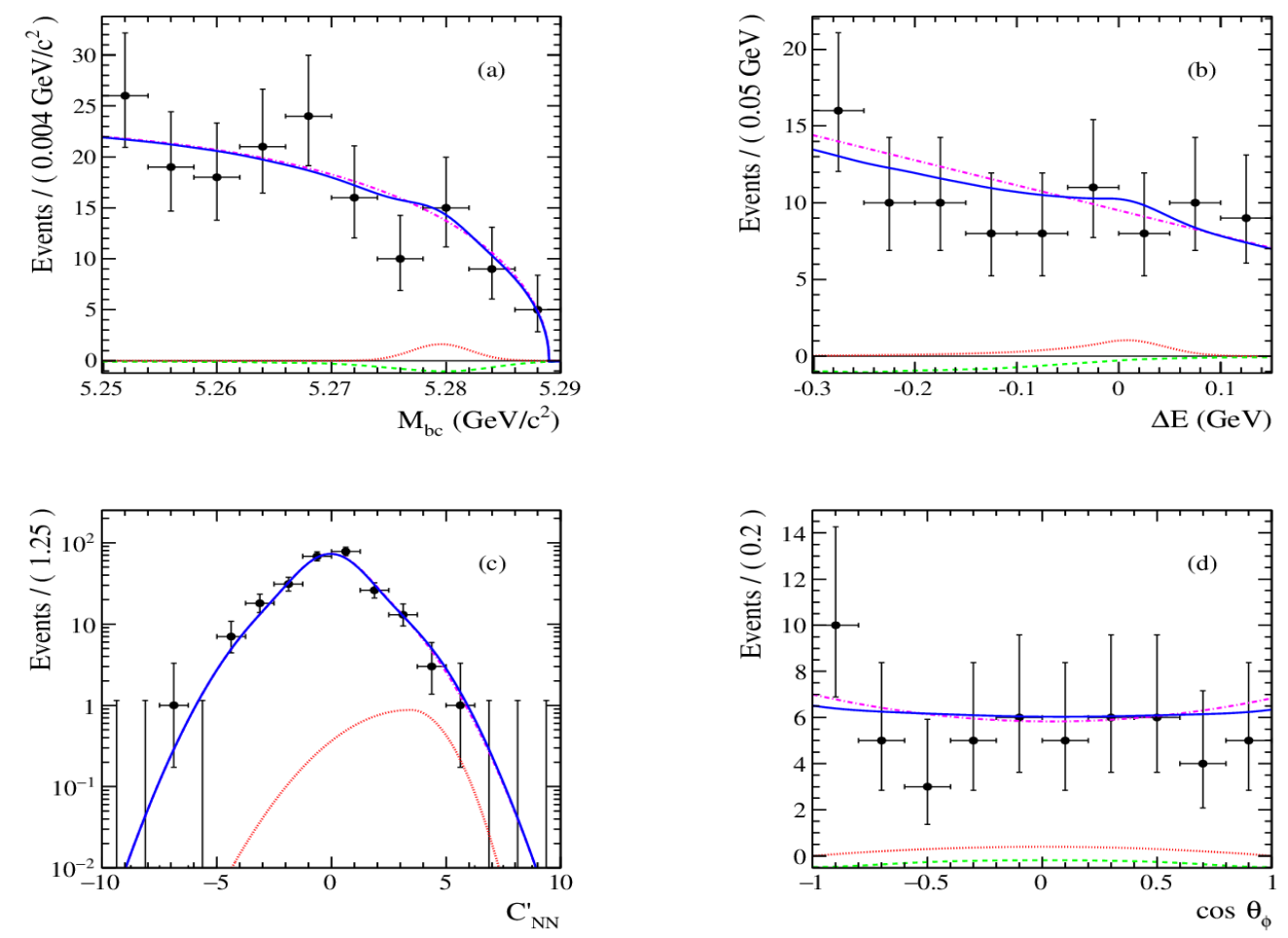

Figure 4: Data projections for the $B^{0} \rightarrow \phi \gamma$ analysis. Points with error bars represent data; the red dotted curves represent signal; the dashed-dotted magenta curves represent continuum events; the dashed green curves represents charmless backgrounds; and the solid blue curves represents the total pdf

SM predictions of $B_{s}^{0} \rightarrow \phi \gamma$ BF lies in the range (3.9 - 4.3) $\times 10^{-5}$ with around $30 \%$ uncertainty [[4]]. First observation of this decay was made by the Belle Collaboration using 23.6 $\mathrm{fb}^{-1} \Upsilon(5 \mathrm{~S})$ data and its BF was measured to be $\left(5.7_{-1.9}^{+2.2}\right) \times 10^{-5}$ [155]. The LHCb Collaboration has estimated its $\mathrm{BF}$ to be $(3.5 \pm 0.4) \times 10^{-5}$ [ [0]]. This analysis done in Belle uses $121 \mathrm{fb}^{-1}$ of $\Upsilon(5 \mathrm{~S})$ data. $B_{s}^{0} \rightarrow \phi \gamma$ mode is reconstructed using various preselection criteria. Analysis and fit procedure is similar to that of the $B^{0} \rightarrow \phi \gamma$ mode. The BF is calculated to be $(3.5 \pm 0.5$ (stat. $) \pm 0.3($ syst. $\left.)+0.6\left(f_{s}\right)\right) \times 10^{-5}$ [ए]]. The fit projections are shown in Figure [5] 

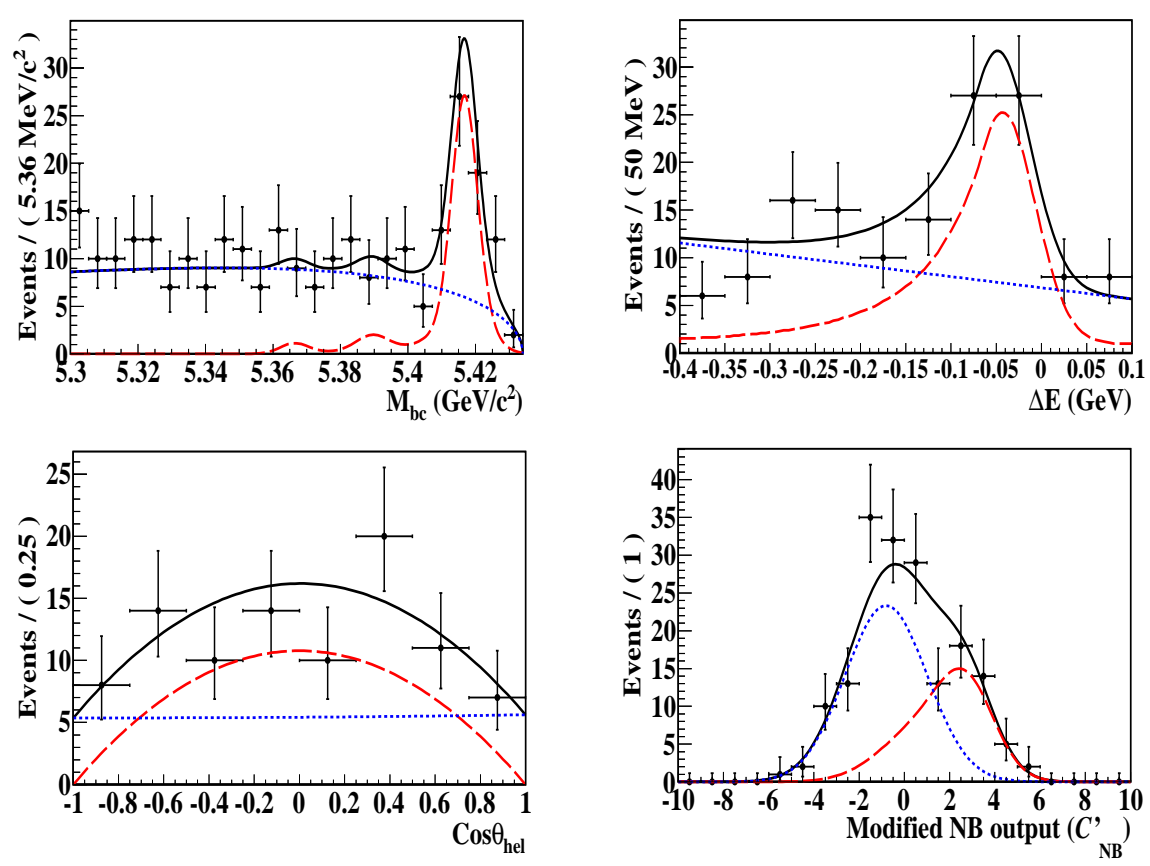

Figure 5: Data fits for the $B_{s}^{0} \rightarrow \phi \gamma$ analysis. Points with error bars represent the data; the solid black curve represents the total fit function; the red dashed (blue dotted) curve represents the signal (continuum background) contribution respectively.

$\mathrm{SM}$ predictions of $B_{s}^{0} \rightarrow \gamma \gamma$ lie in the range of $(0.18-2.45) \times 10^{-6}$ [ए8]. Previous measurement of $B_{s}^{0} \rightarrow \gamma \gamma$ BF provided the $90 \%$ CL UL to be $8.7 \times 10^{-6}$ [प]5]. $B_{s}^{0} \rightarrow \gamma \gamma$ mode is reconstructed and various preselection cuts are applied to reduce the background level. Event shape variables are then fed to NeuroBayes classifier for continuum suppression. A cut is applied on the modified NeuroBayes output $\mathscr{C}_{N B^{\prime}}$. A $2 \mathrm{D}$ fit involving $M_{\mathrm{bc}}$ and $\Delta E$ is done to extract the signal yield. In the $121.4 \mathrm{fb}^{-1} \Upsilon(5 \mathrm{~S})$ data sample no evidence of $B_{s}^{0} \rightarrow \gamma \gamma$ signal is found. The $90 \% \mathrm{CL}$ $\mathrm{UL}$ on its BF is estimated to be $3.1 \times 10^{-6}$ [प]]. The fit projections are shown in Figure. 6 .
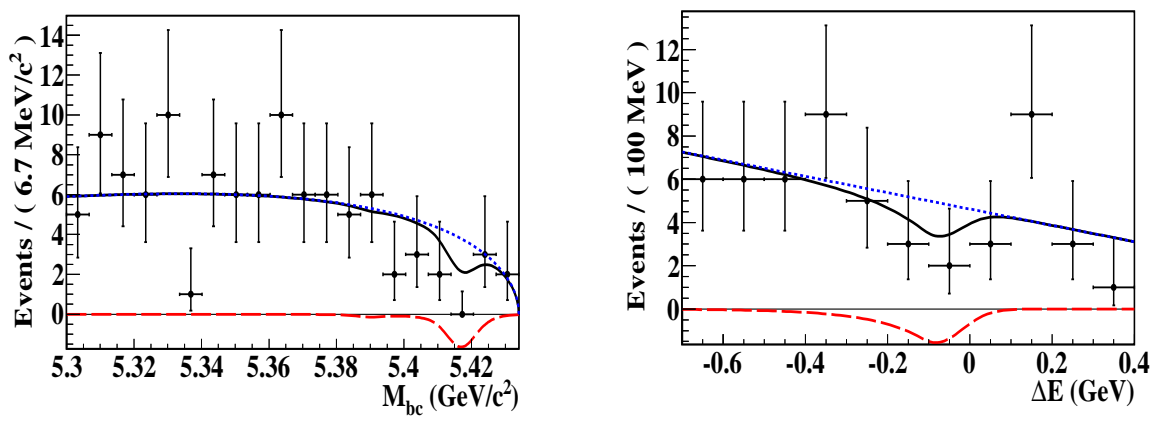

Figure 6: Data fits for the $B_{s}^{0} \rightarrow \gamma \gamma$ analysis. Points with error bars represent the data; the solid black curve represents the total fit function; the red dashed (blue dotted) curve represents the signal (continuum background) contribution respectively. 


\section{Summary}

In this paper we report the results of radiative and annihilation penguin B decays at Belle. Precisely, we discuss the processes proceeding via the $b \rightarrow s$ and $b \rightarrow d$ loop transitions. All the measurements done at Belle are consistent with the SM predictions. The branching fraction of $\bar{B} \rightarrow X_{s} \gamma$ is computed to be $(3.51 \pm 0.17 \pm 0.33) \times 10^{-4}$ for $E_{\gamma}^{*}>1.8 \mathrm{GeV}$ using semi-inclusive approach. CP asymmetry $\left(A_{C P}\right)$ of inclusive $\bar{B} \rightarrow X_{s+d} \gamma$ is measured to be $(2.2 \pm 3.9 \pm 0.9) \%$ for $E_{\gamma}^{*}>2.1 \mathrm{GeV}$ where the leading systematics comes from $B \bar{B}$ background asymmetry. These results are statistically dominant. The branching fraction of the exclusive mode $B_{s}^{0} \rightarrow \phi \gamma$ is calculated to be $(3.5 \pm 0.5$ (stat. $) \pm 0.3($ syst. $\left.) \pm 0.6\left(f_{s}\right)\right) \times 10^{-5}$ and the $90 \%$ CL upper limit of $B^{0} \rightarrow \phi \gamma$ and $B_{s}^{0} \rightarrow \gamma \gamma$ modes are estimated to be $1.0 \times 10^{-7}$ and $3.1 \times 10^{-6}$ respectively.

\section{References}

[1] A. Abashian et al. (Belle Collaboration), Nucl. Instrum. Methods Phys. Res., Sect. A 479, 117 (2002); J. Brodzicka et al. (Belle Collaboration), PTEP 04D001 (2012).

[2] S. Kurokawa and E. Kikutani, Nucl. Instrum. Methods Phys. Res., Sect. A 4991 (2003).

[3] M. Benzke, S. J. Lee, M. Neubert and G. Paz, Phys. Rev. Lett. 106141801 (2011).

[4] T. Hurth, E. Lunghi and W. Porod, Nucl. Phys. B 70456 (2005).

[5] T. E. Coan et al. (CLEO Collaboration), Phys. Rev. Lett. 865661 (2001).

[6] J. P. Lees et al. (Babar Collaboration), Phys. Rev. D 86112008 (2012).

[7] L. Pesántez et al. (Belle Collaboration), Phys. Rev. Lett. 114151601 (2015).

[8] M. Misiak et al., Phys. Rev. Lett. 98022002 (2007); T. Becher and M. Neubert, Phys. Rev. Lett. 98 022003 (2007).

[9] Particle Data Group, Chin. Phys. C 38090001 (2014).

[10] T. Saito et al. (Belle Collaboration), Phys. Rev. D 91052004 (2015).

[11] X. Q. Li, G. R. Lu, R. M. Wang and Y. Yang, Eur. Phys. J. C 3697 (2004); J. Hua, C. Kim and Y. Li, Eur. Phys. J. C 69139 (2010); C. D. Lu, Y. L. Shen and W. Wang, Chin. Phys. Lett. 232684 (2006).

[12] B. Aubert et al. (Babar Collaboration), Phys. Rev. D 72091103 (2005).

[13] Z. King et al. (Belle Collaboration), Phys. Rev. D 93 111101(R) (2016).

[14] A. Ali, B. D. Pecjak, and C. Greub, Eur. Phys. J. C 55577 (2008); P. Ball, G. W. Jones, and R. Zwicky, Phys. Rev. D 75054004 (2007).

[15] J. Wicht et al. (Belle Collaboration), Phys. Rev. Lett. 100121801 (2008).

[16] R. Aaij et al. (LHCb Collaboration), Nucl. Phys. B 8671 (2013).

[17] D. Dutta et al. (Belle Collaboration), Phys. Rev. D $91011101(\mathrm{R})$ (2015).

[18] L. Reina, G. Ricciardi, and A. Soni, Phys. Rev. D 565805 (1997); S. W. Bosch and G. Buchalla, JHEP 2002054 (2002); R. Mohanta and A. Giri, Phys. Rev. D 85014008 (2012). 